\title{
MR Guided Pulsed High Intensity Focused Ultrasound Enhancement of Docetaxel Combined with Radiotherapy for Prostate Cancer Treatment
}

\author{
Lili Chen, Ph.D, Alan Pollack M.D; Ph.D* \\ Department of Radiation Oncology, Fox Chase Cancer Center, Philadelphia, PA 19111-2434 \\ *University of Miami, Miller School of Medicine, Miami, FL 33136
}

\section{Introduction}

This report summarizes our studies based on the funded research project. The project aims were (1) to determine if focused ultrasound (FUS) increases the cellular uptake of ${ }^{3} \mathrm{H}$ docetaxel in vivo, and (2) to establish whether the increased uptake of docetaxel combining with RT will enhance tumor growth inhibition. The sequence of our studies is as follows. First we performed experiments on an acoustic phantom to determine the optimal ultrasonic parameters. Second, we developed techniques for the treatment of prostate tumor-bearing mice. Third, we verified the enhancement of ${ }^{3} \mathrm{H}$-docetaxel uptake in prostate tumors with MRgFUS in vivo. Finally, we investigated the effect of enhancement of docetaxel (Chemotherapy) by MRgFUS on orthotropically implanted prostate tumor in mice in vivo. We summarize the results of these studies in the following sections.

\section{Study on ultrasound output parameters}

Pilot experiments were performed on an MRgFUS system with an acoustic phantom provided by InSightec. The purpose of this study was to determine the ultrasound output including frequency, acoustic power and pulse width that are adequate for the enhancement of chemotherapy in mice, without damage to normal tissues. The MR proton resonance frequency shift sequence was used for temperature mapping during the treatment. We assumed that tissue would not be damaged below $42^{\circ} \mathrm{C}$. Our results suggested that in order to avoid permanent tissue damage, the acoustic power should be equal or less than $5 \mathrm{~W}$. Our results showed that at $1 \mathrm{MHz}$ and an acoustic power of $4 \mathrm{~W}$ the $4{ }^{\circ} \mathrm{C}$ temperature elevation was maintained from 40 joules to 180 joules (sonication time: 10-40 seconds) due to thermal equilibrium. We confirmed that with these ultrasonic parameters there have been no tissue damages (ablation) seen in excised tissues. These phantom measurements provided basic ultrasonic parameters for the MRgFUS system used in our in vivo studies (see below). It should be mentioned that $1 \mathrm{MHz}$ was chosen based on the cavitation mechanism, which is consistent with previous studies (i.e. 1,2).

\section{Tumor models and determination of optimal MR protocols}

All animal studies were carried out under the animal protocol (08-19) approved by IACUC (institutional animal care and use committee) at Fox Chase Cancer Center (FCCC). LNCaP (human prostate cancer) cells $\left(10^{6}\right)$ were injected into the prostates of mice. MRI was performed beginning at 3 weeks after tumor implantation.

In order to use MR guidance for the focused ultrasound treatment, we developed an optimal MR imaging protocol, which allowed us to obtain a higher quality image to visualize the prostate tumor while the scan time remains acceptable for the whole MRgFUS treatment 
procedure within approximately $1 \mathrm{~h}$ anesthesia time.

A quick scan was performed for initial localization using the fast spin echo (FSE) image sequence at low image quality. The scan time was $0.13 \mathrm{~min}$. It was followed by a high quality image sequence with $7 \mathrm{~min}$ scan time in order to identify the tumor target. The MR parameters were: T2-weighted coronal fast spin echo (FSE) sequence; TR/TE=2150/102 ms; Bandwidth: $10.4 \mathrm{kHz}$; FOV=9 x $9 \mathrm{~cm}$; Matrix: 384 x 384 NEX: 4; slice thickness: 2.0 $\mathrm{mm} / 0.0 \mathrm{sp}$; frequency direction: SI and the spatial resolution: 0.23 $\mathrm{mm}$. Based on the coronal image a high quality, axial MR images with 4.25 min was obtained. The total scan time was approximately $11 \mathrm{~min}$. Figure 1 show the high quality image that

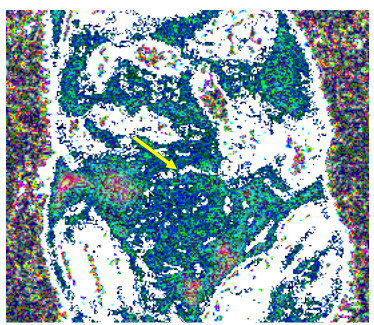

Figure 1. MR images showing the prostate tumor in the coronal view was used for our MRgFUS treatment planning.

\section{Experimental setup}

The mice were treated under general anesthesia. A gel pad was placed on the treatment table in line with the transducer. Degassed water was used for the interface between the treatment table and the gel pad for the acoustic coupling. Care was taken to eliminate any air bubbles between the interfaces. The gel pad was warmed to approximately $37{ }^{\circ} \mathrm{C}$ and a shallow hole measured about $2 \mathrm{~cm} \times 3 \mathrm{~cm}$ with $8 \mathrm{~mm}$ in depth was made. The hole was located in the center on the top of the gel pad and filled with warm degassed water. The animal was carefully placed on the gel pad. The animal stomach was in line with the hole in contract with the degassed water in a prone position. A 3-inch surface coil was placed around the animal to receive the MR signals. A small acoustic phantom was placed beside the animal for the purpose of the ultrasonic beam verification. A plastic glove filled with warm water was placed on the animal to maintain its body temperature. Three localization MR sequences were performed to ensure there were no gas bubbles in the interface between the gel pad and the animal skin (figure

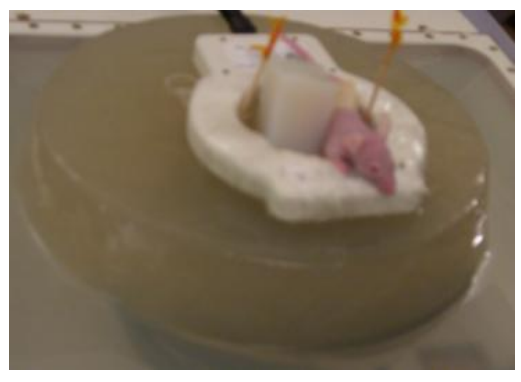

Figure 2. The animal set up for MRgFUS treatment for this project 2).

\section{${ }^{3} \mathrm{H}$-Docetaxel uptake in prostate tumors treated with MRgFUS in vivo}

This study was carried out under the protocols approved by the radiation safety committee and IACUC at FCCC. When prostate tumors reached the size of $160 \pm 10 \mathrm{~mm}^{3}$ on MRI, FUS treatment was preformed using an ExAblate 2000 (InSightec system) with a 1.5 T GE MR scanner. The animals were randomly divided into 3 groups ( $=8$ per group). The 3 groups are: Group 1, HIFU treatment $+{ }^{3} \mathrm{H}$-docetaxel; Group 2, FUS treatment only and Group 3, as control. For group 1, each mouse was treated under general anesthesia with pulsed FUS using $1 \mathrm{MHz} ; 4 \mathrm{~W}$ of acoustic power and the 81 mode setting $(5 \mathrm{~Hz}$ frequency with $0.1 \mathrm{~s}$ power on, $0.1 \mathrm{~s}$ power off) for 60 seconds under MR guidance. Immediately after FUS treatment animal 
received a single dose of i.v injection of docetaxel at $15 \mathrm{mg} / \mathrm{kg}$ mixed with ${ }^{3} \mathrm{H}$-docetaxel at $50 \mathrm{uCi} / \mathrm{kg}$ in $150 \mu \mathrm{l}$. The dose determination was based on the published article (3). Animals in group 2 were treated the same as for group one but without FUS treatment. Animals were sacrificed $30 \mathrm{~min}$ after i.v injections and tumors were removed and processed. The radioactivity of ${ }^{3} \mathrm{H}$-docetaxel in the tumor tissue was quantitatively measured by a liquid scintillation counter.

Our study results showed that all animals tolerated the MRgFUS treatment well. There were no treatment-related adverse events including skin toxicity. Our data show increased ${ }^{3} \mathrm{H}$-docetaxel concentration in tumor in the MRgFUS treated group $(1079 \pm 132 \mathrm{cmp} / 75 \mu \mathrm{g})$ vs. those without MRgFUS treatment $(524 \pm 201 \mathrm{cmp} / 75 \mu \mathrm{g})$ with $\mathrm{P}=0.037$ (figure 3).

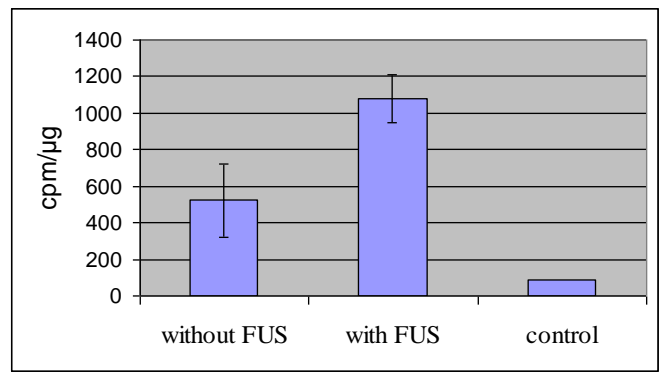

Figure 3. Comparison of ${ }^{3} \mathrm{H}$-Docetaxel between with FUS treatment and without FUS treatment group $(n=8)$.

\section{Effect of Docetaxel with MRgFUS on prostate tumor growth in vivo}

The purpose of this study was to investigate whether or not docetaxel + FUS will improve the prostate tumor control. When tumors reached the volume of $47 \pm 3 \mathrm{~mm}^{3}$ on MRI, treatment was performed. The animals were randomly divided into 3 groups ( $n=5 /$ group). For group 1, mice were treated with MRgFUS once a week for two consecutive weeks. Animals were treated with pulsed ultrasound using $1 \mathrm{MHz} ; 5 \mathrm{~W}$ acoustic power and the 81 mode setting ( $5 \mathrm{~Hz}$ frequency with $0.1 \mathrm{~s}$ power on, $0.1 \mathrm{~s}$ power off) for 60 seconds for one sonication. Multiple sonications (4-6) were used to cover the whole tumor volume. Immediately after the MRgFUS treatment, docetaxel $(10 \mathrm{mg} / \mathrm{kg})$ was injected by tail vein. The animal was allowed to survive for 4 weeks after treatment. The tumor growth was monitored on MRI ( $7 \mathrm{~T}$ ). Animals in Group 2 were treated with docetaxel injection only. Animals in group 3 were treated with FUS only and group 4 was used as control.

Table 1. Comparison of tumor volumes (TV) between the groups $(n=5)$. TX=treatment

\begin{tabular}{|c|c|c|c|c|c|c|}
\hline Groups & $\begin{array}{c}\text { TV }\left(\mathrm{mm}^{3}\right) \\
\text { before } \\
\text { Tx }\end{array}$ & $\begin{array}{c}\text { TV }\left(\mathrm{mm}^{3}\right) 1 \\
\text { wk after Tx }\end{array}$ & $\begin{array}{c}\text { TV }\left(\mathrm{mm}^{3}\right) 4 \\
\text { wks after Tx }\end{array}$ & $\begin{array}{c}\text { Relative TV } \\
\text { before Tx }\end{array}$ & $\begin{array}{c}\text { Relative TV } \\
\text { wk after Tx }\end{array}$ & $\begin{array}{c}\text { Relative TV 4 } \\
\text { wks after Tx }\end{array}$ \\
\hline FUS+Docetaxel & $41.4 \pm 4.0$ & $32.6 \pm 3.2$ & $30.9 \pm 4.60$ & $1.0 \pm 0.11$ & $0.79 \pm 0.05$ & $0.77 \pm 0.1$ \\
\hline Docetaxel & $43.9 \pm 2.8$ & $39.8 \pm 9.9$ & $65.0 \pm 23.9$ & $1.0 \pm 0.06$ & $0.90 \pm 0.21$ & $1.52 \pm 0.6$ \\
\hline FUS & $48.0 \pm 6.2$ & $96.9 \pm 10.3$ & $177.6 \pm 34.4$ & $1.0 \pm 0.13$ & $2.15 \pm 0.5$ & $4.29 \pm 1.3$ \\
\hline Control & $50.0 \pm 3.9$ & $133.0 \pm 17.5$ & $328.5 \pm 54.2$ & $1.0 \pm 0.06$ & $2.69 \pm 0.48$ & $6.77 \pm 1.3$ \\
\hline
\end{tabular}

Our results showed that all animals in groups 3 tolerated FUS well. As expected animals in group 1 and 2 showed weight loss (approximately 15-20\% of the body weight) during chemotherapy and gradually the weight recovered after the treatment. There has been no animal death associated with the treatment. Table 1 summarizes tumor growth before and after treatment for each group. The data was further illustrated in Figure 4. The relative tumor volume in MRgFUS + docetaxel treated group was $0.79 \pm 0.051$ week after and 0.77 
\pm 0.14 weeks after treatment, respectively, while for the group that received only docetaxel it was $0.90 \pm 0.21$ and $1.52 \pm 0.6$ at 1 week and 4 weeks, respectively. Both groups showed smaller tumor volumes compared to the control group. An abstract on the results of this study has been submitted to the AAPM annual meeting (July 2010).

It is interesting to see that the tumor is smaller in the FUS group compared with the control group $(4.3 \pm 1.3$ vs. $6.8 \pm 1.3) 4$ weeks after treatment. Further studies are warranted to investigate the effect of non-thermal FUS on tumor growth control.

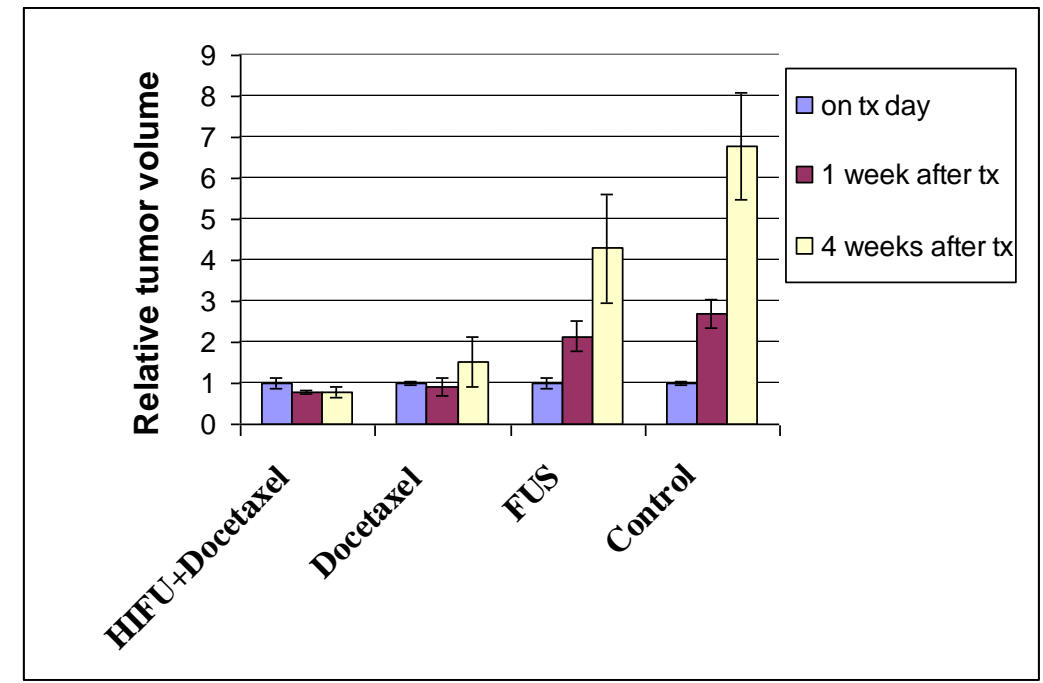

Figure 4. Comparison of relative tumor volume before and after the treatment for each group $(\mathrm{n}=5)$

\section{Discussions}

To study the effect of docetaxel with MRgFUS on prostate tumor in mice in vivo experiments were initially performed using i.p injections with docetaxel. The reason to use i.p injection was based on an assumption that it might not be possible for multiple chemo-drug injections by tail vein in mice although we believed that the i.v injection would be more effective. However, after i.p injections for three consecutive weeks 40-50\% of the animals suffered from severe local GI toxicity showing expanded intestines due to compromised intestinal peristalsis in the docetaxel treated groups. In addition, $75 \%$ of the animals in the group of FUS+docetaxel (i.p) + RT could not tolerate the entire treatment. After we modified our treatment protocol to deliver treatments in 2 consecutive weeks instead of 3 consecutive weeks there were still $17-33 \%$ of animals that could not tolerate the i.p injection. Finally we modified our protocol again to treat with multiple i.v injections and our results showed that animals in the docetaxel groups treated with i.v injections tolerated the treatment well.

Conclusions: Our preliminary results show that MRgFUS has the potential to increase the efficacy of docetaxel in inhibiting prostate cancer growth. 


\section{References}

1. Yuh EL, Shulman SG, Mehta SA, Xie J, Chen L, Frenkel V, Bednarski MD and Li KCP. Delivery of a Systemic Chemotherapeutic Agent to Tumors Using Focused Ultrasound: study in a murine model Radiology 234(2): 431 - 437 (2005)

2. Dittmar KM, Xie J, Hunter F, Trimble C, Bur M, Frenkel V, Li KC. Pulsed high-intensity focused ultrasound enhances systemic administration of naked DNA in squamous cell carcinoma model: initial experience. Radiology 235(2):541-6, (2005)

3. Engels FK, Buijs, D, Loos WJ et al. Quantification of $\left[{ }^{3} \mathrm{H}\right]-$ docetaxel in faeces and urine: development and validation of a novel combustion method. Anticancer Drugs. 17: 63-67 (2006)

\section{Published proceedings and abstracts}

1) L Chen, Z Mu, P Hachem, L Chung, T Richardson, C Ma, A Pollack. MR Guided Focused Ultrasound (MRgFUS) for Treatment of Prostate Cancer: Feasibility Study of Increasing Intratumoral Uptake of Docetaxel in Vivo. Medical Physics: 35: 2876, 2008

2) L Chen, Z Mu, P Hachem, C-M Ma, A Pollack. Enhancement of Drug Delivery in Prostate Tumor in vivo Using MR Guided Focused Ultrasound (MRgHIFU). WC, IFMBE 25: 341-344, 2009

\section{Papers in preparation}

1) L Chen, Zhaomei Mu, Hachem Paul, C-M Ma and Alan Pollack MR. Guided Focused Ultrasound for the Treatment of Prostate Cancer: A Feasibility Study of Increasing Intratumoral Uptake of ${ }^{3} \mathrm{H}$-Docetaxel in vivo

2) L Chen, Z Mu, A Wallentine, A Pollack and C-M Charlie Ma. Synergetic Effect of Docetaxel and MR Guided Focused Ultrasound (MRgFUS) for Prostate Cancer in vivo 